\title{
An Improvement of the Asymptotic Iteration Method for Exactly Solvable Eigenvalue Problems*
}

\author{
I. Boztosun ${ }^{\dagger}$, M. Karakoc \\ Faculty of Arts and Sciences, Department of Physics, \\ Erciyes University, Kayseri, Turkey
}

(Dated: (Received 27 July 2007))

\begin{abstract}
We derive a formula that simplifies the original asymptotic iteration method formulation to find the energy eigenvalues for the analytically solvable cases. We then show that there is a connection between the asymptotic iteration and the Nikiforov-Uvarov methods, which both solve the second order linear ordinary differential equations analytically.
\end{abstract}

PACS numbers: 03.65.Ge

\footnotetext{
* Supported by the Scientific and Technical Research Council of Turkey (TÜBİTAK) under Grant No TBAG-106T024, Erciyes University (EÜBAP-FBA-04-02), and Turkish Academy of Sciences (TÜBAGEBIP).

† Email: boztosun@erciyes.edu.tr
} 
Analytical solutions of the radial Schrödinger equation are of high importance in quantum mechanics since the wave function contains all the necessary information to describe a quantum system fully ${ }^{[1-12]}$. The asymptotic iteration method (AIM) $)^{[1,2]}$ and the NikiforovUvarov method $^{[3]}$ are two different approaches to solve the resulting Schrödinger equation and they have been successfully applied to a wide variety of problems ${ }^{[4-14]}$.

In this work, we derive a formula which simplifies the original AIM formulation to find the energy eigenvalues for the analytically solvable cases. This formulation shows that there is a connection between the AIM and Nikiforov-Uvarov methods regarding the solution of differential equations.

$\mathrm{AIM}^{[1]}$ is proposed to solve the second-order differential equations in the form

$$
y^{\prime \prime}=\lambda_{0}(r) y^{\prime}+s_{0}(r) y
$$

where $\lambda_{0}(r) \neq 0$ and $\mathrm{s}_{0}(\mathrm{r}), \lambda_{0}(\mathrm{r})$ are in $\mathrm{C}_{\infty}(\mathrm{a}, \mathrm{b})$. Eq. (1) as given in Ref. [1] has a general solution

$$
y(r)=\exp \left(-\int^{r} \alpha\left(r^{\prime}\right) d r^{\prime}\right)\left[C_{2}+C_{1} \int^{r} \exp \left(\int^{r^{\prime}}\left(\lambda_{0}\left(r^{\prime \prime}\right)+2 \alpha\left(r^{\prime \prime}\right)\right) d r^{\prime \prime}\right) d r^{\prime}\right],
$$

if $k>0$, for sufficiently large $k, \alpha(r)$ values can be obtained by

$$
\frac{s_{k}(r)}{\lambda_{k}(r)}=\frac{s_{k-1}(r)}{\lambda_{k-1}(r)}=\alpha(r)
$$

where

$$
\begin{aligned}
& \lambda_{k}(r)=\lambda_{k-1}^{\prime}(r)+s_{k-1}(r)+\lambda_{0}(r) \lambda_{k-1}(r), \\
& s_{k}(r)=s_{k-1}^{\prime}(r)+s_{0}(r) \lambda_{k-1}(r), \quad k=1,2,3, \cdots
\end{aligned}
$$

In this method, the idea is to convert the radial Schrödinger equation into the form of Eq. (1) for a given potential. Then, $\mathrm{s}_{0}(r)$ and $\lambda_{0}(r)$ are determined, and $\mathrm{s}_{k}(r)$ and $\lambda_{k}(r)$ parameters are calculated. The energy eigenvalues are determined by the quantization condition given by the equation:

$$
\delta_{k}(r)=\lambda_{k}(r) s_{k-1}(r)-\lambda_{k-1}(r) s_{k}(r)=0, \quad k=1,2,3, \cdots .
$$

If we rearrange Eq. (1) by introducing new forms for $\lambda_{0}(r)$ and $s_{0}(r)$ as follows:

$$
\lambda_{0}(r)=-\frac{\tau(r)}{\sigma(r)}, \quad s_{0}(r)=-\frac{\gamma_{n}}{\sigma(r)}
$$


where $\tau$ and $\sigma$ are new functions and $\gamma_{n}$ is a constant with which we quantize the energy. Inserting them into Eq. (1), the second-order differential equation takes the form

$$
y^{\prime \prime}=-\frac{\tau(r)}{\sigma(r)} y^{\prime}-\frac{\gamma_{n}}{\sigma(r)} y
$$

An essential ingredient of AIM is the quantization condition given by Eq. (5). Every particular value in Eq. (5) corresponds to an energy eigenvalue. Therefore, if we first take $k=1$ and $n=0$, put them into Eq. (6) and then use Eq. (4) for $\gamma_{0} \neq 0$, the quantization condition given by Eq. (5) reduces to

$$
\tau^{\prime}(r)+\gamma_{0}=0
$$

which should hold for all $r$. This means that $\tau(r)$ is a polynomial of the first degree at most. If we choose now $n=1$ and $k=2$, using Eq. (4), the quantization condition given by Eq. (5) reduces to

$$
\left[\gamma_{1}+\tau^{\prime}(r)\right]\left[\left(\gamma_{1}+2 \tau^{\prime}(r)+\sigma^{\prime \prime}(r)\right)-\tau^{\prime \prime}(r)\left(\tau(r)+\sigma^{\prime}(r)\right)\right]=0
$$

which should also hold for all $r$. Since we have already established that $\tau(r)$ is a polynomial of the first degree at most, thus, $\tau^{\prime \prime}(r)=0$. Assuming the spectrum of our problem to be non-degenerate, i.e. $\gamma_{1} \neq \gamma_{0}$, and taking into account Eq. (8), we conclude that $\sigma^{\prime \prime}(r)$ is a polynomial of the second degree at most. In such a case, Eq. (7) and, hence, Eq. (1) are of hypergeometric type (We point out that the comments in this part have been made by an unanimous referee of the Phys. Lett. A for another paper of ours).

Already knowing the properties of $\tau(r)$ and $\sigma(r)$ and by using AIM procedure given by Eq. (4), we can calculate $\lambda_{n}(r)$ and $s_{n}(r)$ as follows, in order to formulate the energy eigenvalue equation,

$$
\begin{aligned}
& \lambda_{1}(r)=\frac{\gamma\left(\tau(r)+\sigma^{\prime}(r)\right)}{\sigma(r)^{2}}, \quad s_{1}(r)=\frac{\tau(r)\left(\tau(r)+\sigma^{\prime}(r)\right)-\sigma(r)\left(\gamma+\tau^{\prime}(r)\right)}{\sigma(r)^{2}}, \\
& \ldots \text { etc }
\end{aligned}
$$

If we use the termination condition of AIM given by Eq. (5), the energy eigenvalues are 
obtained as follows

$$
\begin{aligned}
\frac{s_{0}}{\lambda_{0}} & =\frac{s_{1}}{\lambda_{1}} \Rightarrow \gamma_{0}=0 \\
\frac{s_{1}}{\lambda_{1}} & =\frac{s_{2}}{\lambda_{2}} \Rightarrow \gamma_{1}=-\tau^{\prime}(r) \\
\frac{s_{2}}{\lambda_{2}} & =\frac{s_{3}}{\lambda_{3}} \Rightarrow \gamma_{2}=-2 \tau^{\prime}(r)-\sigma^{\prime \prime}(r) \\
\frac{s_{3}}{\lambda_{3}} & =\frac{s_{4}}{\lambda_{4}} \Rightarrow \gamma_{3}=-3 \tau^{\prime}(r)-3 \sigma^{\prime \prime}(r), \\
\ldots \text { etc } &
\end{aligned}
$$

which can be generalized as

$$
\gamma_{n}=-n \tau^{\prime}(r)-\frac{n(n-1)}{2} \sigma^{\prime \prime}(r)
$$

This formula gives directly the energy eigenvalues for differential equation (7). It replaces the iterative calculations needed to find the energy eigenvalues in the original AIM formulation. Also, it is exactly the same as the Nikiforov-Uvarov energy eigenvalue equation. This is briefly derived as follows:

The Nikiforov-Uvarov equation is written as

$$
\psi(r)^{\prime \prime}+\frac{\tau(r)}{\sigma(r)} \psi^{\prime}(r)+\frac{\tilde{\sigma}(r)}{\sigma^{2}(r)} \psi(r)=0,
$$

where $\sigma(r)$ and $\tilde{\sigma}(r)$ are the polynomials of the second-degree at most, and $\tilde{\tau}(r)$ is a first-degree polynomial ${ }^{[3,4,6-9]}$. Hence, from Eq. (16), the Schrödinger equation or the Schrödinger-like equations can be solved analytically by this method. In order to find a particular solution of Eq. (16), the following transformation is used:

$$
\psi(r)=\phi(r) y(r)
$$

It reduces Eq. (16) to an equation of hypergeometric type,

$$
\sigma(r) y^{\prime \prime}+\tau(r) y^{\prime}+\gamma y=0
$$

and $\phi(r)$ is defined as a logarithmic derivative in the following form and its solutions can be obtained from

$$
\phi^{\prime}(r) / \phi(r)=\pi(r) / \sigma(r)
$$

The function $\pi$ and the parameter $\lambda$ required for this method are defined as follows:

$$
\pi(r)=\frac{\sigma^{\prime}-\tau}{2} \pm \sqrt{\left(\frac{\sigma^{\prime}-\tau}{2}\right)^{2}-\tilde{\sigma}+k \sigma}
$$




$$
\lambda=k+\pi^{\prime} .
$$

On the other hand, in order to find the value of $k$, the expression under the square root must be the square of a polynomial. Thus, the eigenvalue equation for the Schrödinger equation becomes

$$
\lambda_{n}=-n \tau^{\prime}-\frac{n(n-1)}{2} \sigma^{\prime \prime} .
$$

Next, we present some applications. For classical differential equations, in order to show the applicability of the above formula, let us consider the classical differential equations which give polynomial solutions ${ }^{[15]}$. The results are presented in Table 1.

For some other applications reported previously in the literature by using AIM, we may take the following examples:

In the Morse potential case, if we take the transformed equation (31) of Ref. [16] as

$$
\frac{d^{2} f_{n}(r)}{d r^{2}}=\left(\frac{2 \beta y-2 \varepsilon-\alpha}{\alpha r}\right) \frac{d f_{n}(r)}{d r}+\left(\frac{2 \varepsilon \beta+\alpha \beta-2 \beta^{2}}{r \alpha^{2}}\right) f_{n}(r),
$$

where

$$
-\tau(r)=2 \beta r-2 \varepsilon-\alpha, \quad \sigma=\alpha r, \quad-\gamma_{n}=\left(\frac{2 \varepsilon \beta+\alpha \beta-2 \beta^{2}}{\alpha}\right) .
$$

Using Eq. (15), the eigenvalues turn out to be

$$
\varepsilon_{n}=\beta-\left(n+\frac{1}{2}\right) \alpha \quad n=0,1,2,3, \cdots
$$

For the deformed Hùlten potential case, if we take the transformed equation, Eq. (18) of Ref. [16] can be rewritten as

$$
\frac{d^{2} f(r)}{d r^{2}}=\left[\frac{(2 \varepsilon+3) q r-(2 \varepsilon+1)}{r(1-q r)}\right] \frac{d f(r)}{d r}+\left[\frac{2 \varepsilon q+q-\beta^{2}}{r(1-q r)}\right] f(r) .
$$

If we compare this equation with Eq. (6), we obtain the functions

$$
-\tau(r)=(2 \varepsilon+3) q r-(2 \varepsilon+1), \quad \sigma(r)=-r(1-q r), \quad-\gamma_{n}=2 \varepsilon q+q-\beta^{2} .
$$

Using Eq. (15), the eigenvalues turn out to be

$$
\varepsilon_{n}=-\frac{1}{(2 n+2)}\left(\frac{q(n+1)^{2}-\beta^{2}}{q}\right), \quad n=0,1,2,3, \cdots .
$$

In the Kratzer potential case, if we take the transformed equation, Eq. (14) of Ref. [17] can be rewritten as 


$$
f^{\prime \prime}(r)=2\left(\varepsilon-\frac{(\Lambda+1)}{r}\right) f^{\prime}(r)+\left(\frac{2(\Lambda+1) \varepsilon-A}{r}\right) f(r) .
$$

If we compare this equation with Eq. (6), we obtain the functions

$$
-\tau(r)=2(\varepsilon r-(\Lambda+1)), \quad \sigma=r, \quad-\gamma_{n}=2(\Lambda+1) \varepsilon-A
$$

Using Eq. (15), the eigenvalues turn out to be

$$
\varepsilon_{n}=-\frac{1}{(2 n+2)}\left(\frac{q(n+1)^{2}-\beta^{2}}{q}\right), \quad n=0,1,2,3, \cdots
$$

Next, we discuss the eigenfunctions. If we insert Eq. (15) into Eq. (7) and use Eqs. (2) and (3), we can obtain the wave functions as follows:

$$
\begin{aligned}
& y_{0}(r)=1, \\
& y_{1}(r)=\tau, \\
& y_{2}(r)=\tau^{2}+\tau \sigma^{\prime}+\tau^{\prime} \sigma+\sigma \sigma^{\prime \prime}, \\
& y_{3}(r)=\tau^{3}+3 \tau^{2} \sigma^{\prime}+2 \tau \sigma^{\prime 2}+3 \tau \tau^{\prime} \sigma+4 \tau^{\prime} \sigma \sigma^{\prime}+5 \tau \sigma \sigma^{\prime \prime}+6 \sigma \sigma^{\prime} \sigma^{\prime \prime}, \\
& \ldots .
\end{aligned}
$$

These iterative functions can be generalized in a closed form as follows:

$$
y_{n}(r)=\frac{1}{\rho(r)} \frac{d^{n}}{d s^{n}}\left[\sigma(r)^{n} \rho(r)\right], \quad n=0,1,2,3, \cdots
$$

where $\rho(r)$ has to satisfy ${ }^{[3]}$

$$
[\sigma(r) \rho(r)]^{\prime}=\tau(r) \rho(r)
$$

If we apply Eq. (36) to the deformed Hulten potential case as an example, we obtain

$$
\begin{aligned}
y_{0}(r)= & 1 \\
y_{1}(r)= & \left(2 \varepsilon_{1}+3\right) q r-\left(2 \varepsilon_{1}+1\right), \\
y_{2}(r)= & 2\left[\left(2 \varepsilon_{2}^{2}+3 \varepsilon_{2}\right)+\left(\varepsilon_{2}+2\right)\left(2 \varepsilon_{2}+5\right) q^{2} r^{2}-4\left(\varepsilon_{2}+1\right)\left(\varepsilon_{2}+2\right) q r+1\right], \\
y_{3}(r)= & 2\left(\varepsilon_{3}+3\right)\left(2 \varepsilon_{3}+5\right)\left(2 \varepsilon_{3}+7\right) q^{3} r^{3}-6\left(\varepsilon_{3}+3\right)\left(2 \varepsilon_{3}+3\right)\left(2 \varepsilon_{3}+5\right) q^{2} r^{2}+ \\
& +2\left(3 \varepsilon_{3}\left(4 \varepsilon_{3}\left(\varepsilon_{3}+5\right)+31\right)+45\right) q r-2\left(\varepsilon_{3}\left(4 \varepsilon_{3}\left(\varepsilon_{3}+3\right)+11\right)+3\right),
\end{aligned}
$$


These equations can be written in a generalized way as a hypergeometric function,

$$
\left.y_{n}(r)=(-1)^{n} \frac{\Gamma\left(2 \varepsilon_{n}+n+1\right)}{\Gamma\left(2 \varepsilon_{n}+1\right)}\right)_{2} F_{1}\left(-n,\left(2 \varepsilon_{n}+n+2\right) ;\left\{2 \varepsilon_{n}+1\right\} ; q r\right) .
$$

If Eq. (42) is compared with the results of Ref. [16], it can be seen that they are exactly the same eigenfunctions.

In summary, we have derived a formula for the analytically solvable potentials, which is given in Eq. (15). This formula replaces the iterative calculations and reduces the calculation workload in the original AIM formulation to find the energy eigenvalues. We have also shown that there is a close connection between the AIM and the Nikiforov-Uvarov methods to solve differential equations.

[1] Ciftci H, Hall R L and Saad N 2003 J. Phys. A: Math. Gen. 3611807

[2] Ciftci H, Hall R L and Saad N 2005 J. Phys. A: Math. Gen. 381147

[3] Nikiforov A F and Uvarov V B 1988 Special Functions of Mathematical Physics (Basel: Birkhäuser)

[4] Cheng Y F and Dai T Q 2007 Physica Scripta 75274

[5] Gonul B and Koksal K 2007 Physica Scripta 75686

[6] Egrifes H, Demirhan D and Büyükkilic F 1999 Physica Scripta 5990

[7] Aktas M and Sever R 2005 J. Phys. Math. Chem. 37139

[8] Yasuk F, Berkdemir C and Berkdemir A 2005 J. Phys. A: Math. Gen. 386579

[9] Yasuk F, Durmus A and Boztosun I 2006 J. Math. Phys. 47082302

[10] Boztosun I, Karakoc M, Yasuk F and Durmus A 2006 J. Math. Phys. 47062301

[11] Bayrak O and Boztosun I 2006 J. Phys. A: Math. Gen. 396955

[12] Aygun M, Bayrak O and Boztosun I 2007 J. Phys. B: At. Mol. Opt. Phys. 40537

[13] Fernández F M 2004 J. Phys. A: Math. Gen. 376173

[14] Barakat T 2006 J. Phys. A: Math. Gen. 39823

[15] Saad N, Hall R L and Ciftci H 2006 J. Phys. A: Math. Gen. 3913445

[16] Bayrak O and Boztosun I 2006 J. Mol. Struct.: Theochem. 80217

[17] Bayrak O, Boztosun I and Ciftci H 2007 Int. J. Quantum Chem. 107540 


\begin{tabular}{|c|c|c|c|c|}
\hline Differential equation & $-\tau$ & $\sigma$ & $-\gamma_{n}$ & Eigenvalue \\
\hline Cauchy-Euler ${ }^{1}$ & $\alpha(r-b)$ & $(r-a)^{2}$ & $\beta$ & $\beta=n(n-1-\alpha)$ \\
\hline Hermite $^{2 a}$ & $2 r$ & 1 & $-2 k$ & $k=n$ \\
\hline Hermite $^{2 b}$ & $a r+b$ & 1 & $c$ & $c=-n a$ \\
\hline Laguerre & $r-1$ & $r$ & $a$ & $a=-n$ \\
\hline Confluent $^{3}$ & $b r-c$ & $r$ & $a$ & $a=-n b$ \\
\hline Hypergeometric & $(a+b+1) r-c$ & $r(1-r)$ & $a b$ & $a=-n$ or $b=-n$ \\
\hline Legendre & $-2 r$ & $r^{2}-1$ & $m(m+1)$ & $m=n$ \\
\hline Jacobi & $(\alpha+\beta+2) r+\beta+\alpha$ & $1-r^{2}$ & $-\gamma$ & $\gamma=n(n+\alpha+\beta+1)$ \\
\hline Chebyshev $^{4 a}$ & $r$ & $1-r^{2}$ & $-m$ & $m=n^{2}$ \\
\hline Chebyshev $^{4 b}$ & $3 r$ & $1-r^{2}$ & $-m$ & $m=n(n+2)$ \\
\hline Gegenbauer & $(1+2 k) r$ & $\left(1-r^{2}\right)$ & $-\lambda$ & $\lambda=n(n+2 k)$ \\
\hline Hyperspherical & $2(1+k) r$ & $\left(1-r^{2}\right)$ & $-\lambda$ & $\lambda=n(n+1+2 k)$ \\
\hline Bessel $^{5 a}$ & $-2(r+1)$ & $r^{2}$ & $\gamma$ & $\gamma=n(n+1)$ \\
\hline Generalized Bessel $^{5 b}$ & $-(a r+b)$ & $r^{2}$ & $\gamma$ & $\gamma=n(n+a-1)$ \\
\hline
\end{tabular}

TABLE I: Application of Eq. (15) to classical differential equations which give polynomial solutions. 